\section{Auriculoterapia como tratamento na melhoria da qualidade de vida dos servidores do CRAS de Blumenau-SC: estudo piloto}

\section{Auriculotherapy as a treatment to improve the life quality of the workers of CRAS in Blumenau-SC: pilot study}

\section{RESUMO}

Introdução: Qualidade de vida reflete perspectivas individuais e coletivas, sendo influenciada pelos aspectos socioeconômicos, psicológicos e pelas experiências de cada indivíduo. Dessa forma, buscar práticas alternativas e complementares que auxiliem na manutenção dessa qualidade é de suma importância. Objetivo: Avaliar a efetividade desta prática na melhoria da qualidade de vida dos servidores do Centro de Referências de Assistência Social (CRAS) do município de Blumenau-SC. Material e métodos: A coleta de dados foi realizada a partir da aplicação de dois questionários antes e após a realização do tratamento, a Versão Brasileira do Questionário de Qualidade de Vida - SF-36 e o Teste de Lipp - ISS. Foram realizadas 5 sessões de auriculoterapia com cada servidor. Os resultados de cada paciente foram analisados individualmente por meio de gráficos e discutidos entre si. Resultados: Ao analisar os resultados individuais de cada paciente, utilizando o Questionário de Qualidade de Vida - SF-36, percebe-se que $80 \%$ dos servidores apresentaram melhora em 4 ou mais dos 8 domínios. $O$ paciente 1,3 e 5 apresentarem melhora em $50 \%$ dos domínios. $O$ paciente 4 apresentou melhora somente em 2 domínios e o paciente 2 foi o que obteve o efeito mais expressivo, melhora em 6 dos 8 domínios. Os resultados encontrados ao fim do tratamento foram positivos, tanto quando se analisou os pacientes individualmente quanto em relação a cada domínio do Questionário de Qualidade de Vida - SF-36. Conclusões: Qualidade de vida reflete perspectivas individuais e coletivas, sendo influenciada pelos aspectos socioeconômicos, psicológicos e pelas experiências de cada indivíduo. Dessa forma, buscar práticas complementares que auxiliem na manutenção dessa qualidade é de suma importância.

Palavras-chave: Qualidade de vida. CRAS. Acupuntura auricular
Júlia Dresch Devilla

- Biomédica

- Departamento de Farmácia

- Centro de Ciências da Saúde

- Universidade Regional de Blumenau - FURB

- Blumenau-SC

- juliadevilla@hotmail.com

orcid.org/0000-0002-9216-9844

Milene Dinah Faht

- Farmacêutica e Psicóloga

- Centro de Referências de Assistência

Social (CRAS) III Fortaleza

- Prefeitura de Blumenau - Blumenau-SC

-milenefaht@yahoo.com.br

orcid.org/0000-0003-0681-3478

Murilo Luiz Cerutti

- Farmacêutico e Professor

- Departamento de Medicina - Centro de Ciências da Saúde

- Universidade Regional de Blumenau - FURB

- Blumenau-SC

muriloceruttineuro@gmail.com - orcid.org/0000-0002-2625-7782

Caio Mauricio Mendes de Cordova

- Farmacêutico e Professor

- Departamento de Farmácia - Centro de Ciências da Saúde

- Universidade Regional de Blumenau - FURB

- Blumenau-SC

- cmcordova@furb.br

- orcid.org/0000-0001-6090-0367

Caroline Valente

- Farmacêutica, Acupunturista e Professora

- Departamento de Ciências Naturais

- Centro de Ciências Exatas e Naturais

- Universidade Regional de Blumenau - FURB

- Blumenau-SC

carolvalente11@gmail.com

- orcid.org/0000-0002-3816-3871

DOI: $10.19177 /$ cntc.v8e15201917-25

Recebido: 26/10/2020

Aprovado: 07/12/2020 


\begin{abstract}
Introduction: Quality of life reflects individual and collectives perspectives, being influenced by socioeconomics and psychological aspects besides the experiences of each individual. This way, search for alternative and complementary practices who help in maintenance of this quality is very import. Objective: To evaluate the effectiveness of this practice in improving the life quality of the workers of the Social Assistance Reference Center (CRAS) in the city of Blumenau, SC, Brazil. Material and methods: Data collection was carried out by applying two questionnaires before and after the treatment, the Brazilian version of the Quality of Life Questionnaire - SF-36 and the Lipp Test - ISS. Five auriculotherapy sessions were held with each worker. The results of each patient were examined individually using graphs and discussed among them. Results: When the individual results of each patient were examined by using the Quality of Life Questionnaire - SF-36, it could be seen that $80 \%$ of the workers improved in 4 or more of the 8 domains. Patients 1,3 , and 5 improved in $50 \%$ of the domains. Patient 4 showed improved in only 2 domains and patient 2 had the most expressive effect, improvement in 6 of 8 domains. The results found at the end of the treatment were positive, both when the patients were examined individually and in relation to each domain of the Quality of Life Questionnaire - SF-36. Conclusions: The life quality reflects individual and collective perspectives, being influenced by socioeconomic and psychological aspects, and by the experiences of each individual. Thus, seeking complementary practices that help maintain this quality is of paramount importance.
\end{abstract}

Keywords. Quality of life. CRAS. Auricular acupuncture

\title{
INTRODUÇÃO
}

A Medicina Tradicional Chinesa (MTC) é uma das mais antigas formas terapêuticas. Tem como princípio o entendimento holístico do mundo, analisando o corpo humano na sua integridade ${ }^{1}$. A MTC busca tratar o indivíduo como um todo, em sua complexidade, promovendo diagnósticos prévios e utilizando um conjunto de técnicas orientais como recurso terapêutico².

A auriculoterapia, prática integrante da MTC, se baseia na utilização da orelha para diagnóstico e tratamento de diversas enfermidades. $O$ pavilhão auricular apresenta pontos correspondentes a todos os órgãos e partes do corpo, podendo demonstrar todas suas alterações fisiológicas ou desequilíbrios orgânicos ${ }^{3}$. Atualmente, existem duas escolas de auriculoterapia, a chinesa que se baseia nas teorias da MTC e a francesa que é fundamentada pela neurofisiologia. Ambas as escolas apresentaram resultados positivos, o que se comprova pela verificação da sua eficácia por meio de pesquisas e credibilidade que apresenta no mundo todo, sendo a terapia de microssistema mais utilizada ${ }^{4}$.

O tratamento nesta prática é feito pela estimulação dos pontos da orelha por meio de agulhas, esferas ou sementes de mostarda, o que promove estabilidade interna do organismo ${ }^{5}$. A realização da auriculoterapia estimula o próprio organismo a produzir substâncias necessárias para normalizar seu funcionamento. Seu resultado é tão seguro que hoje em dia vem sendo muito utilizada no tratamento de dores, obesidade, tabagismo, ansiedade, depressão, entre outras patologias ${ }^{6}$.

A Qualidade de Vida se caracteriza pela percepção do indivíduo de sua posição no contexto cultural, no sistema de valores em que está inserido e em relação aos seus objetivos, padrões, expectativas e preocupações. O que demonstra que diversos fatores a influenciam, sendo o trabalho um grande determinante. Como diversos fatores influenciam a Qualidade de Vida, assim como o trabalho, cada vez mais tem se investido em pesquisa sobre o tema. Analisa-se como os trabalhadores se sentem em relação ao seu ambiente de trabalho e à sua ocupação; refere-se, então, a aspectos psicológicos, físicos e ambientais associados a profissão ${ }^{7}$. A rotina de trabalho pode gerar muito desgaste físico e emocional. Diversos fatores podem levar a isso, como os baixos salários, muitas horas de trabalho, grande demanda de atendimento para pou- 
ca equipe, falta de expectativa de crescimento, entre outros. A soma de todas essas condições acaba afetando a qualidade de vida de todos esses profissionais, em diferentes intensidades.

$\mathrm{O}$ estresse se encontra presente no cotidiano de pelo menos $90 \%$ da população mundial. Hoje em dia é reconhecido que o estresse laboral é um dos principais problemas para a saúde dos trabalhadores e para o bom funcionamento dos locais onde trabalham. Segundo a OMS, níveis altos desse estresse são considerados prejudicais à saúde psicológica e física dos profissionais, refletindo negativamente na sua produtividade. Baseado nisso, um trabalhador estressado se torna mais suscetível ao desenvolvimento de patologias e menos motivado, improdutivo e inseguro em suas realizações ${ }^{8}$.

Tendo em vista os prejuízos que o estresse pode causar ao trabalhador, têm-se gerado um grande interesse por parte de empresas e da sociedade pela busca de métodos eficazes para sua redução. Medidas que consigam diminuir os seus níveis e aliviar a tensão e ansiedade se tornam importantes para que o trabalho passe a ser uma fonte de auto realização, satisfação e manutenção de relações pessoais produtivas e saudáveis. Partindo disso, cuidar dos profissionais que oferecem serviços de saúde se torna de suma importância, visto que um bom atendimento aos pacientes depende, principalmente, de equipes de trabalho saudáveis ${ }^{9-11}$.

As práticas integrativas e complementares (PICs) têm sido cada vez mais utilizadas como forma de tratamento. Essas práticas têm se mostrado de grande eficácia para tratar muitas enfermidades, além de trazerem uma melhoria na qualidade de vida. A auriculoterapia tem ganhado mais espaço e reconhecimento na área das PICs. As principais vantagens dessa prática são: não é invasiva, pode ser realizada com a utilização de sementes de mostarda e microesferas, é de fácil administração e eficaz no tratamento de diversas patologias, além de apresentar resultados rápidos e baixo custo. Pode ainda ser aplicada como complemento a outras formas de tratamento.

Esse estudo tem como objetivo, através de um estudo piloto, avaliar a efetividade da auriculoterapia na melhoria da qualidade de vida dos servidores do Centro de Referências de Assistência Social (CRAS) III Fortaleza do município de Blumenau-SC.

\section{MATERIAL E MÉTODOS}

O presente estudo foi realizado no Centro de Referência de Assistência Social (CRAS) III Fortaleza, localizado na Rua Alberto Felício Adriano ${ }^{\circ}$ 150, Fortaleza, município de Blumenau, Santa Catarina. Os CRAS são unidades públicas responsáveis por fornecer serviços de assistência social, principalmente, nas áreas com maior vulnerabilidade e riscos sociais dos municípios.

A coleta de dados foi feita a partir da aplicação da Versão Brasileira do Questionário de Qualidade de Vida - SF-36 e do Teste de Lipp ISS - Inventário Sintomas de Estresse antes e após a realização do tratamento, com o objetivo de avaliar se houve alguma melhora. $\mathrm{O}$ tratamento foi feito em 5 sessões, intercalando a orelha direita e esquerda a cada sessão e sendo realizadas uma vez por semana. Os pontos utilizados foram Shen Men, Rim, Tronco Nervoso Cerebral, Coração, Fígado, Ansiedade e Tensão com reforço atrás, Ápice da Orelha e San Jiao (Figura 1). A estimulação dos pontos foi feita com sementes de mostardas, por ser uma forma não invasiva de tratamento.

Figura 1 - Mapa dos pontos de auriculoterapia utilizados no tratamento.

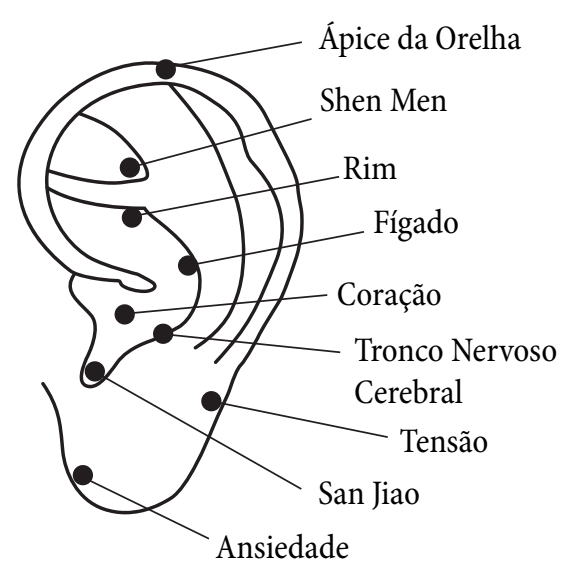

O projeto iniciou com um total de seis pacientes, na segunda sessão mais um servidor aceitou participar, somando um total de sete 
participantes. Porém, na sessão seguinte dois desistiram e apenas cinco concluíram a pesquisa. O único critério para participação na pesquisa era ser servidor do CRAS.

Após o término do tratamento, os resultados obtidos foram tabelas e analisados individualmente, devido ao número pequeno de participantes. Após isso, foram analisados entre si e com resultados obtidos em pesquisas semelhantes encontradas na literatura.

O projeto foi aprovação no dia 27 de abril de 2017 pelo Comitê de Ética em Pesquisa em Seres Humanos da Fundação Universidade Regional de Blumenau-FURB(CAAE:64977517.8.0000.5370) e todos os participantes assinaram o Termo de Consentimento Livre e Esclarecido, permitindo que seus resultados fossem utilizados na pesquisa.

\section{RESULTADOS}

A pesquisa foi realizada com um total de $5 \mathrm{pa}$ cientes, sendo todos do sexo feminino. Na primeira sessão de atendimento com auriculoterapia cada paciente relatou quais eram suas principais queixas. Na figura 2 pode ser observado o resultado da primeira anamnese demonstrando as principais queixas e a porcentagem de pacientes que as relataram.

Figura 2 - Principais queixas relatadas pelos pacientes na primeira sessão de tratamento.

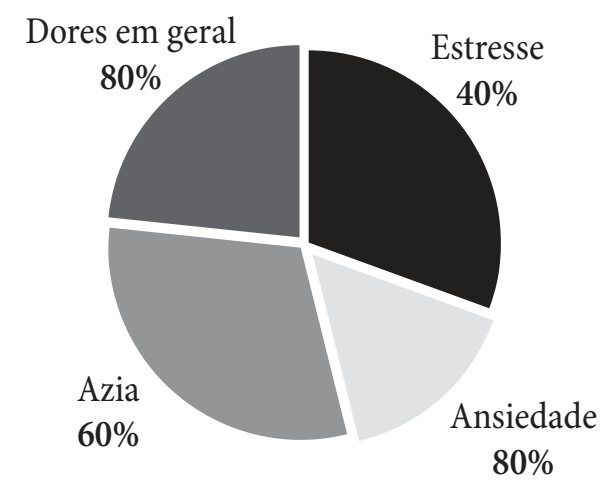

Em todas as sessões de atendimento foi solicitado aos pacientes que descrevessem como se sentiam em relação a sessão passada. A partir disso eles relatavam se haviam se sentido me- lhor ou não e se sentiram algo diferente. Abaixo está o relato individual de cada paciente:

Paciente 1 - Após a primeira sessão, na segunda e terceira sessão a paciente relatou se sentir normal com uma melhora geral dos sintomas. Nas duas últimas sessões relatou melhoria da memória e do estresse, com melhoria da concentração e facilidade para estudar;

Paciente 2 - Em todas as sessões a paciente relatou se sentir mais focada, centrada em suas atividades, porém em algumas semanas se sentia mais agitada ou irritada devido situações associadas ao dia-a-dia; Paciente 3 - Na segunda sessão a paciente relatou dor no estômago, cansaço físico e mental e bastante dor no ponto do Rim. Já na terceira descreveu melhora do cansaço que apresentava, porém problemas intestinais, que melhoraram após a quarta sessão com a troca do pavilhão auricular. E na última sessão relatou aumento da sede e compulsão alimentar, com melhora do estresse;

Paciente 4 - A paciente relatou em todas as sessões após os pontos terem sido colocados na orelha direita melhora da insônia, ou seja, na segunda e quarta sessão. Nas demais relatou não ter dormido muito bem, o que reflete o fato da paciente responder de forma melhor ao tratamento na orelha direita;

Paciente 5 - Nas primeiras sessões, relatou não sentir nenhuma diferença. Mas assim como a paciente 4 , sentiu alterações diferentes quando trocava o pavilhão auricular. Quando os pontos eram colocados no pavilhão esquerda sentia aumento da frequência e volume urinários, o que melhorava na sessão seguinte.

\section{Teste de Lipp - ISS}

O Teste de Lipp - ISS é utilizado para avaliar se o paciente possui algum sintoma de estresse ou mesmo se está propenso a ter. Este teste é divido em 3 fases: Fase de Alerta, que consiste no período de contato e adaptação do organismo a fonte de estresse, é identificada pela presença de 7 ou mais sintomas descritos no teste nas últimas 24 horas; Fase de Resistência, é quando o organismo tenta reestabelecer o equilíbrio e eliminar o agente estressante, identificada pela presença de 4 ou mais 
sintomas no último mês; e Fase de Exaustão, ocorre quando o organismo retorna a fase de alerta, porém acompanhada de doenças, é identificada pela presença de 9 ou mais sintomas nos últimos 3 meses. $\mathrm{O}$ teste foi aplicado antes do início do tratamento e após seu término e seus resultados estão apresentadas na tabela 1 .

\section{Tabela 1 - Resultados do Teste de Lipp - ISS}

\begin{tabular}{c|cc}
\hline & \multicolumn{1}{c}{$\begin{array}{c}\text { Início do } \\
\text { Tratamento }\end{array}$} & $\begin{array}{c}\text { Término do } \\
\text { Tratamento }\end{array}$ \\
\hline Paciente 1 & Fase de resitência & Fase de resitência \\
\hline Paciente 2 & Fase de resitência & \\
\hline Paciente 3 & Fase de exaustão & Fase de exaustão \\
\hline Paciente 4 & Fase de resitência & \\
\hline Paciente 5 & Fase de resitência & Fase de resitência \\
\hline
\end{tabular}

\section{Questionário Qualidade de Vida - SF36}

O SF36 apresenta seus resultados separados em 8 domínios, os valores variam de 0 a 100, sendo 0 a pior condição e 100 a melhor. Esses domínios devem ser analisados separadamente para que se possa avaliar se houve alguma melhora ou não. A partir disso, em cada gráfico apresentado abaixo foram analisados separadamente cada paciente (Figura 3 a 7). Em cada domínio há os resultados dos questionários respondidos no início e no término do tratamento.

Figura 3 - Resultados SF-36 - Paciente 1

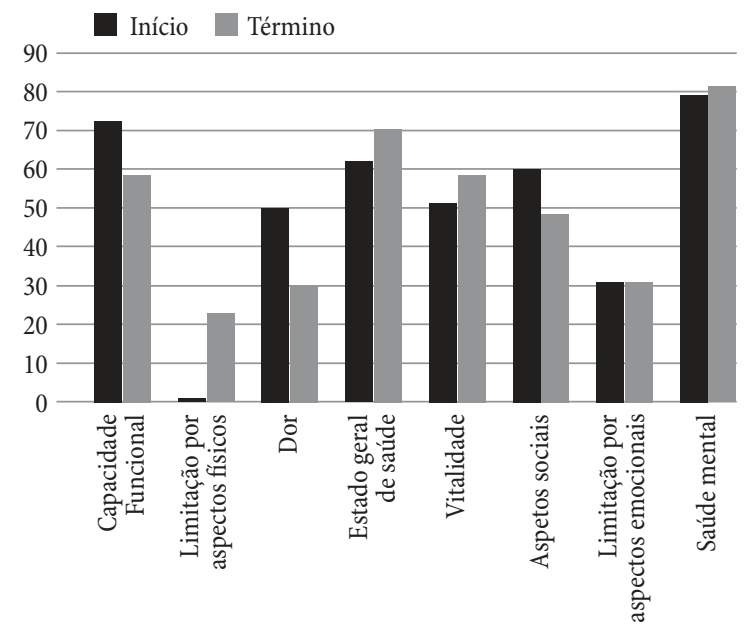

O Paciente 1 apresentou melhora em 50\% dos domínios, sendo eles limitação por aspectos físicos, estado geral de saúde, vitalidade e saúde mental. Um domínio permaneceu com a mesma pontuação e 3 apresentaram diminuição.

Figura 4 - Resultados SF-36 - Paciente 2

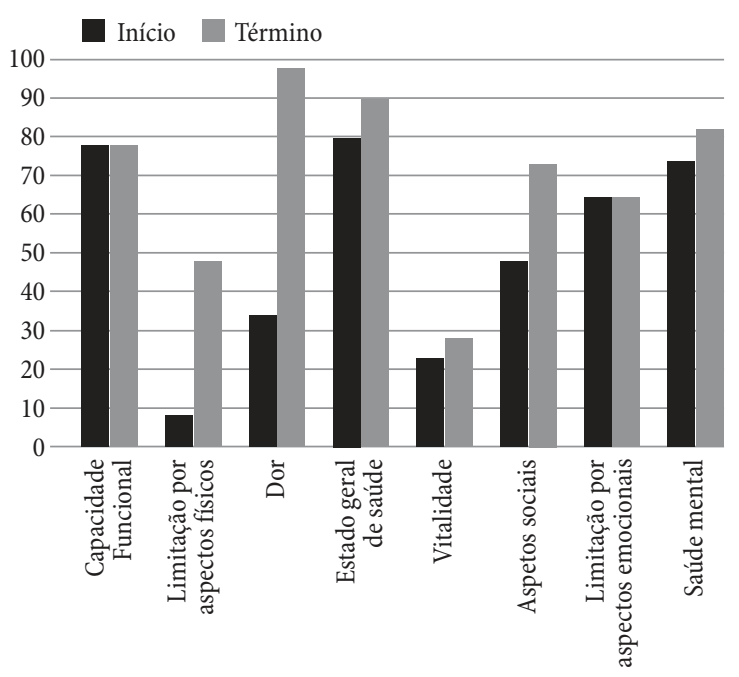

O Paciente 2 apresentou melhora em 6 dos 8 domínios e permaneceu com a mesma pontuação em 2, sendo eles capacidade funcional e limitação por aspectos emocionais.

Figura 5 - Resultados SF-36 - Paciente 3

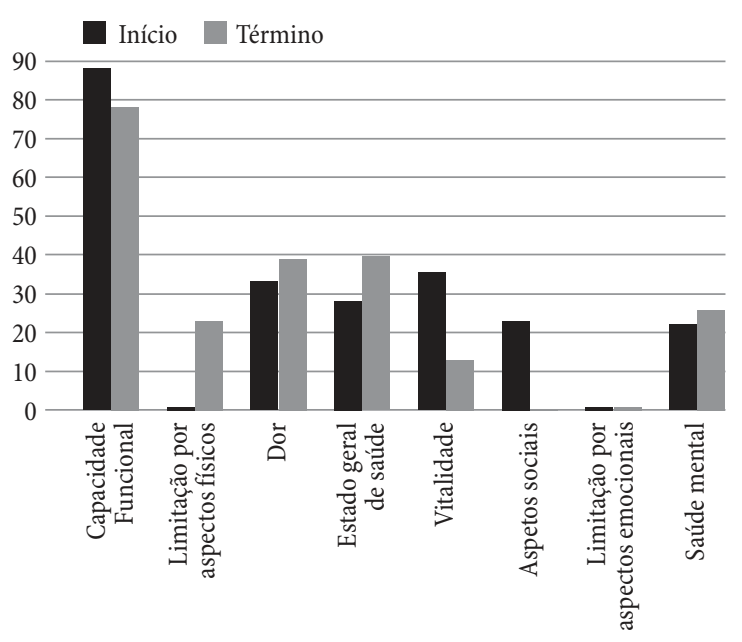

O Paciente 3 apresentou melhora em 50\% dos domínios, sendo eles limitação por aspectos físicos, dor, estado geral de saúde e saúde mental. Em 3 domínios houve diminuição da pontuação e 1 permaneceu com pontuação mínima. 
Figura 6 - Resultados SF-36 - Paciente 4

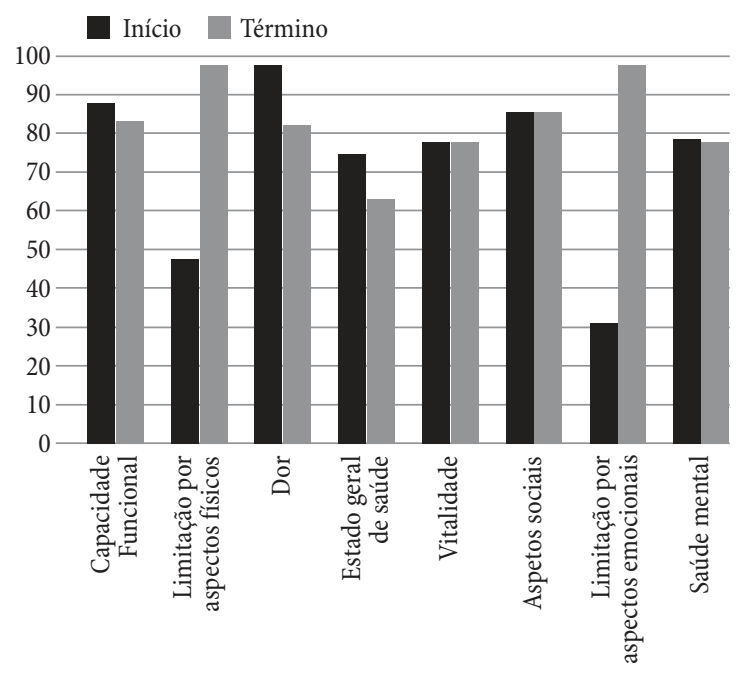

O Paciente 4 apresentou melhora em apenas 2 domínios, sendo eles limitação por aspectos físicos e por aspectos emocionais. Em 2 domínios sua pontuação permaneceu mínima e nos demais houve diminuição.

Figura 7 - Resultados SF-36 - Paciente 5

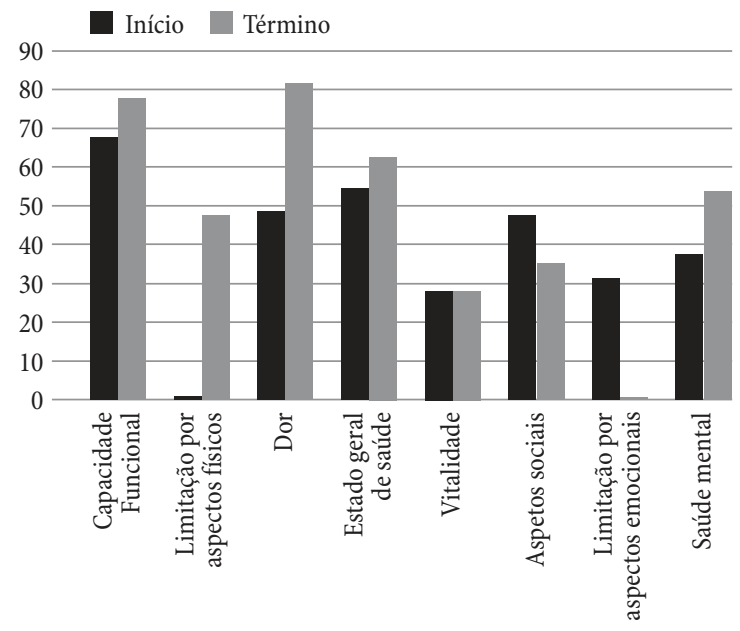

O Paciente 5 apresentou melhora em mais de $50 \%$ dos domínios e 1 permaneceu com a mesma pontuação. Apenas 2 domínios apresentaram diminuição da pontuação, sendo eles aspectos sociais e limitação por aspectos emocionais.

\section{DISCUSSÃO}

Qualidade de vida reflete perspectivas individuais e coletivas, sendo influenciada pelos aspectos socioeconômicos, psicológicos e pelas experiências de cada indivíduo. Dessa forma, buscar práticas alter- nativas e complementares que auxiliem na manutenção dessa qualidade é de suma importância. A auriculoterapia é uma prática que se baseia na utilização do pavilhão auricular para diagnóstico e tratamento de diversas enfermidades.

Normalmente a auriculoterapia é iniciada pela colação dos pontos do "triângulo cibernético", composto pelo Shen Men, Rim e Tronco Nervoso Cerebral ${ }^{9-13}$. O ponto Shen Men, primeiro ponto a ser colocado, é utilizado como ponto sedante, anti-inflamatório, analgésico e para acalmar a mente. O Rim, um dos principais pontos de auriculoterapia, é indicado para disfunções ósseas e articulares, infertilidade, medo, insegurança, pânico e perda de memória recente. O Tronco Nervoso Cerebral é um ponto relaxante indicado para acalmar a mente, convulsão e síndrome do pânico ${ }^{4,14}$.

Os pontos Shen Men, Rim e Tronco Nervoso Cerebral foram utilizados em todas as pesquisas analisadas $^{9-13,15-17}$. Maior parte dos estudos usaram o triângulo cibernético completo com o objetivo de tratar especificamente o estresse. Porém, alguns que tinham como objetivo individualizar o tratamento ou identificar se a auriculoterapia seria mais eficiente quando realizada com protocolo ou sem incluíram outros pontos auriculares, que complementariam o tratamento.

A partir da análise dos resultados do Teste de Lipp - ISS percebe-se que $80 \%$ dos pacientes apresentavam-se na fase de resistência antes do início do tratamento. Destes $80 \%, 40 \%$ permaneceram na mesma fase e os outros $40 \%$ não marcaram sintomas suficiente para se enquadrar em nenhuma fase de estresse após a realização da auriculoterapia. $\mathrm{O}$ único paciente que se apresentava na fase de exaustão antes do tratamento permaneceu na mesma fase após o tratamento.

Como os sintomas da fase de resistência e da fase de exaustão são relacionados ao último mês e aos últimos três meses, respectivamente, dificulta a interpretação da real eficácia do tratamento, pois esse foi realizado em 5 sessões (1 mês e uma semana). Sendo assim, para o teste 
de Lipp - ISS, estudos com um número maior de paciente se faz necessário para demonstrar que a auriculoterapia é eficaz na melhora dos sintomas do estresse.

Bettiol $^{18}$ (2010) realizou uma pesquisa para avaliar os efeitos da auriculoterapia sobre o estresse em acadêmicos de fisioterapia. Para avaliação do estresse foi utilizado o Inventário de Sintomas de Estresse para Adultos de Lipp ISSL, o qual foi aplicado antes do início do tratamento e após o término do mesmo. O questionário empregado é semelhante ao utilizado na presente pesquisa e foi aplicado da mesma forma. Os resultados apresentados pelas duas pesquisas foram semelhantes, apesar das populações dos estudos serem diferentes. Em ambas, maior parte dos pacientes apresentavam-se na fase de resistência, no início do tratamento, e após a realização da auriculoterapia houve uma diminuição dos sinais e sintomas do estresse.

Em relação ao Questionário de Qualidade de Vida - SF-36. Ao analisar os resultados individuais percebe-se que $80 \%$ dos servidores apresentaram melhora em 4 ou mais domínios. Dessa forma, podemos perceber que, ao comparar todos os pacientes em conjunto, houve uma melhora da qualidade de vida em relação ao início do tratamento.

Quando analisados os domínios separadamente percebemos que nos domínios capacidade funcional e aspectos sociais identifica-se que não houve uma melhora, pois em ambos apenas um paciente apresentou melhora, um permaneceu igual e os demais pioraram. Nas limitações por aspectos emocionais não houve melhora relevante, apenas um apresentou melhora e dois permaneceram igual. Na saúde mental dois pacientes melhoraram e apenas um piorou. Na dor houve melhora sendo que apenas dois pacientes pioraram. Nos domínios limitações por aspectos físicos e estado geral de saúde houve melhora bem relevante, no primeiro todos os pacientes apresentaram melhora e no segundo apenas um piorou.
A estimulação dos pontos auriculares pode ser realizada de diversas formas, com a utilização de agulhas intradérmicas, sementes de mostarda ou microesferas. Algumas pesquisas demonstram que o tratamento quando realizado com agulhas apresenta resultados mais rápido do que com a semente de mostarda. A aplicação de sementes de mostarda tem como vantagem ser uma forma de tratamento não invasiva, que pode ser utilizada com mais facilidade em crianças. Porém, depende da estimulação dos pontos pelo paciente para seu funcionamento, quando esta é realizada de forma incorreta o tratamento pode não ser tão eficaz ${ }^{19,12}$.

Kurebayashi ${ }^{9,10,12}$ realizou estudos para verificar os níveis de estresse de profissionais de enfermagem e identificar se os resultados seriam melhores para utilização de sementes de mostarda ou para agulhas semipermanentes. A amostra foi dividida em três grupos, um grupo controle (sem intervenção) e dois grupos com intervenção, um com sementes e outro com agulha. Foram realizadas 8 sessões, uma por semana e os pontos utilizados foram Shen Men, Rim e Tronco Nervoso Cerebral. Os profissionais apresentaram nível médio $(58,7 \%)$ e nível alto de estresse $(41,3 \%)$ e a auriculoterapia foi eficaz na redução do estresse, principalmente nos com níveis mais elevados. $\mathrm{O}$ tratamento realizado com agulhas apresentou resultados mais rápido e consequentemente mais positivos do que o grupo que utilizou sementes ${ }^{9,10,12}$.

Kurebayashi e Silva ${ }^{13}$ (2014) realizaram uma pesquisa com profissionais de enfermagem para avaliar se a auriculoterapia com protocolo ou sem protocolo seria mais eficaz na redução dos níveis de estresse, levando em consideração o nível de estresse e se o paciente apresentava morbidades prévias. Ambos os grupos receberam 12 sessões, duas por semana, com colocação de agulhas semipermanentes. Maior parte dos pacientes com morbidades estavam no grupo sem protocolo e foram esses que conseguiram resultados positivos na redução do estresse. 
O grupo protocolo, por ser voltado para o tratamento apenas do estresse, foi eficaz no tratamento de pessoas saudáveis apenas. $O$ fato de os resultados terem sido melhores para o grupo sem protocolo demonstra que a auriculoterapia apresenta resultados mais positivos quando realizada de forma individualizada, principalmente em pacientes que já apresente morbidades prévias.

Segundo estudo realizado por Figueiredo ${ }^{20}$ (2017), os enfermeiros estão expostos a um ambiente estressante, principalmente pelo fato de serem responsáveis por cuidar do outro. Muitas vezes tem sua vida dedicada ao trabalho, limitando seu tempo livre para lazer, sono e até mesmo alimentação, o que acaba tornando-os estressados. Por isso a necessidade de cuidar desses profissionais que quando estressados, com a qualidade de vida comprometida, podem cuidar dos pacientes de forma inadequada. Em seu estudo, a autora encontrou resultados muito positivos na utilização da auriculoterapia como tratamento para melhora da qualidade de vida.

Com esse estudo, podemos observar que a auriculoterapia é eficaz como tratamento complementar na melhora da qualidade de vida. Porém, como a amostra foi pequena seria necessário realizar novas pesquisas com um número maior de participantes.

\section{CONTRIBUIÇÃO DOS AUTORES}

Contribuição específica de cada autor para o artigo.

1. Concepção, projeto, análise e interpretação dos dados: Júlia Dresch Devilla, Milene Dinah Faht, Murilo Luiz Cerutti, Caio Mauricio Mendes de Cordova e Caroline Valente

2. Redação do artigo e revisão crítica relevante do conteúdo intelectual: Murilo Luiz Cerutti, Caio Mauricio Mendes de Cordova e Caroline Valente

3. Aprovação final da versão a ser publicada: Júlia Dresch Devilla, Milene Dinah Faht, Murilo Luiz Cerutti, Caio Mauricio Mendes de Cordova e Caroline Valente

\section{AGRADECIMENTOS}

Agradecemos à coordenação e servidores do Centro de Referências de Assistência Social (CRAS) III Fortaleza do município de Blumenau-SC.
A partir do trabalho realizado, podemos concluir que a auriculoterapia tem grande potencial como tratamento complementar na melhora da qualidade de vida. Porém, como a amostra foi pequena seria necessário realizar novas pesquisas com um número maior de participantes para confirmar a real eficácia. Além disso, pelo fato de ser uma prática complementar a outros tratamentos, diversos fatores podem influenciar no seu efeito final. Individualizar o tratamento, colocando pontos específicos para os problemas de cada paciente provavelmente traria resultados ainda mais positivos.

Atualmente, as pesquisas estão direcionando esforços na descoberta de novas terapias que possuam baixo custo, reduzam ou diminuíam efeitos colaterais, que possam ser mais naturais e obtenham resultados satisfatórios. Dessa forma, buscar práticas alternativas e complementares, como auriculoterapia, que fazem parte da PNPIC-SUS e que auxiliam na manutenção da qualidade de vida é de suma importância.

Por fim, como existem poucas publicações investigando o uso destas práticas, gostaríamos de ressaltar, que trabalhos como esses poderão inocentar a pesquisa e as discussões com as auriculoterapia.

\section{CONSIDERAÇÕES FINAIS} PICs ampliando ainda mais o conhecimento da 


\section{CONFLITOS DE INTERESSE}

Não há conflito de interesses.

\section{FONTES DE FINANCIAMENTO}

\section{FURB}

\section{REFERÊNCIAS}

1. He YH e Ne ZB. Teoria Básica da Medicina Tradicional Chinesa. São Paulo: Atheneu; 1999.

2. Lu A, Jia H, Xiao C, Lu Q. Theory of traditional Chinese medicine and therapeutic method of diseases. World Journal of Gastroenterology. 2004; 10(13): 1854-1856.

3. Lee EW. Aurículo Acupuntura. São Paulo: Ícone; 2010.

4. Neves ML. Manual Prático de Auriculoterapia. Porto Alegre: Merithus; 2014.

5. Enomoto J. Auriculoterapia Oriental: Método Enomóto. São Paulo: Ícone; 2015.

6. Domingo SJJ. Auriculoterapia. [Monografia de Especialização]. Matinhos: Universidade Federal do Paraná; 2011.

7. Nespeca M e Cyrillo DC. Qualidade de vida no trabalho de funcionários públicos: papel da nutrição e da qualidade de vida. Acta Scientiarum. Health Sciences.2011; 33(2): 187-195.

8. World Health Organization (WHO). General guidelines for methodologies on research and evaluation of traditional medicine. Geneva: WHO. 2000.

9. Kurebayashi LFS, Gnatta JR, Borges TP, DA Silva MJP. Aplicabilidade da auriculoterapia para reduzir estresse e como estratégia de coping em profissionais de enfermagem. Rev. Latino-Am. Enfermagem. 2012a; 20(5): [08 telas].

10. Kurebayashi LFS, Gnatta JR, Borges TP, Belisse G, Coca S, Minami A, Souza TM, Da Silva MJP. Aplicabilidade da auriculoterapia com agulhas ou sementes para diminuição de estresse em profissionais de enfermagem. Rev Esc Enferm USP. 2012b; 46(1): 89-95.

11. Kurebayashi LFS, Gnatta JR, Borges TP, Da Silva MJP. Eficácia da auriculoterapia para estresse segundo experiência do terapeuta: ensaio clínico. Acta Paul. Enferm. 2012c; 25(5): 694-700.
12. Kurebayashi LFS, Gnatta JR, Borges TP, Da Silva MJP. Avaliação diagnóstica da Medicina Tradicional Chinesa dos sintomas de estresse tratados pela auriculoterapia: ensaio clínico. Rev. Eletr. Enf. 2014; 16(1): 68-76.

13. Kurebayashi LFS e Da Silva MJP. Eficácia da auriculoterapia chinesa para o estresse em equipe de enfermagem: ensaio clínico randomizado. Rev. Latino-Am. Enfermagem. 2014; 22(3): 371-8.

14. Fonseca WP. Acupuntura auricular chinesa. São Paulo: Andreoli; 2013.

15. Clemente LA, Salvi JO, Souza LMT. A efetividade da Auriculoterapia no tratamentodo estresse e da Síndrome de Burnout em professores universitários. Cadernos de Naturologia e Terapias Complementares. 2015; 4(7): 21-27.

16. Kurebayashi LFS e Da Silva MJP. Auriculoterapia Chinesa para melhoria de qualidade de vida de equipe de Enfermagem. Rev Bras Enferm. 2015; 68(1): 117-23.

17. Cruz LMTS e Salvi JO. A auriculoterapia cibernética em idosas: relato de caso. Cadernos de Naturologia e Terapias Complementares. 2016; 5(9): 63-68.

18. Bettiol LG. Análise dos efeitos da auriculoterapia sobre o estresse em acadêmicos dos três últimos semestres de graduação de Fisioterapia da UNESC. [TCC de Graduação]. Criciúma: Universidade do Extremo Sul Catarinense; 2010.

19. Lopes GS. Auriculoterapia no combate ao estresse em professores universitários: estudo piloto. [Dissertação de Mestrado]. Manaus: Universidade Federal do Amazonas; 2016.

20. Figueiredo SN. Auriculoterapia para redução do estresse ocupacional e melhoria da qualidade de vida em enfermagem na amazônia: ensaio clínico randomizado. [Dissertação de Mestrado]. Manaus: Universidade do Estado do Amazonas; 2017. 